\title{
Liver transplantation in the era of pandemic COVID-19
}

\author{
Ramlah RAMLAH*
}

Faculty of Biology, Universitas Gadjah Mada, Indonesia

Introduction: Liver transplantation is considered the ultimate solution for patients with end-stage chronic liver disease. Patients with liver transplant need special care starting from preoperative preparation, surgical intervention ending with postoperative care. The effect of coronavirus disease 2019 (COVID-19) on liver transplantation programmes and recipients is still not completely understood but overall involves the risk of donor-derived transmission, health-care resource utilization and the effect of immunosuppression. The aim of this study is to describe the situation of liver transplantation in the era of pandemic COVID-19.

Methods: Data obtained from secondary data on 13 articles journal evaluated by searching in PubMed, EMBASE, and the Cochrane Library database that have been carried out in the last 2 years (2019-2020).

Results: The result showed that patients with liver disease need special attention and continuous follow-up. Similarly, transplant candidates also need special care. Restricting non-transplant elective surgical procedures, managing transplant patients in separate outpatient clinics, and in-patient wards can prevent transmission of infection both to patients and healthcare workers. Telemedicine can help in the triage of patients to screen for symptoms of COVID-19 before their regular appointment. Patients with novel COVID-19 experience various degrees of liver function abnormalities. Liver injury requires extensive work-up and continuous surveillance and can be multifactorial and heterogeneous in nature.

Conclusions: It can be concluded that in management of immunosuppressive therapy and drug-drug interactions in liver transplant recipients infected with COVID-19 should be cautiously practiced to prevent rejection and effectively treat the underlying infection. 